\title{
Handgrip dynamometry: a surrogate marker of malnutrition to predict the prognosis in alcoholic liver disease
}

\author{
Nitin Rangrao Gaikwad, Sudhir Jagdishprasad Gupta, Amol Rajendra Samarth, \\ Tushar Hiralal Sankalecha
}

Government Medical College and Super Specialty Hospital, Nagpur, Maharashtra, India

\section{Abstract}

Background The aim of the study was to determine the utility of handgrip dynamometry (HGD) in predicting short term mortality and complications in alcoholic liver disease.

Methods Patients with alcoholic liver disease were included and nutritional assessment was done using the Subjective Global Assessment (SGA), HGD and other conventional parameters. Mortality rates and complications were compared to nutritional status.

Results 80 patients were included in the study. Mean age of patients was $43.06 \pm 10.03$ years. 69 patients survived and 11 patients died within the 3 month study duration. Handgrip strength (HGS) was higher in SGA A $(28.76 \pm 5.48 \mathrm{~kg})$ than SGA B $(22.43 \pm 4.95 \mathrm{~kg})$ and SGA C $(16.78 \pm 3.83 \mathrm{~kg})(\mathrm{P}=<0.001)$. Number of complications including spontaneous bacterial Peritonitis, gastrointestinal bleeding and encephalopathy in SGA C group were $66.66 \%$, in SGA B $20.75 \%$ and SGA A $10 \%$. Mean HGS was significantly higher in the survivors $(24.23 \pm 5.86)$ compared to nonsurvivors $(18.04 \pm 4.82)(\mathrm{P}=0.0011)$. There was a strong negative correlation between the HGS and Child-Pugh score $(\mathrm{P}=<0.0012)$. Multivariate logistic regression analysis to assess the risk factors for death showed handgrip to be in the suggestive significance range $(\mathrm{P}=0.072)$. The sensitivity of HGS was $88.41 \%$ in predicting short term mortality.

Conclusions HGS correlates with Child-Pugh score in predicting short term mortality. HGD is a simple, inexpensive and sensitive tool for assessing the nutritional status in alcoholic liver disease and can reliably predict its complications and survival.

Keywords Handgrip dynamometry, nutritional status, mortality

Ann Gastroenterol 2016; 29 (3): 1-6

\section{Introduction}

Cirrhosis has many complications such as ascites, infections, gastrointestinal bleeding and encephalopathy; however sarcopenia, a frequently unseen complication is also common, reflecting the poor nutritional status in cirrhotics which adversely impacts the survival, quality of life, and response to stressors such as infections and surgery.

Department of Gastroenterology, Government Medical College and Super Specialty Hospital, Nagpur, Maharashtra, India

Conflict of Interest: None

Correspondence to: Sudhir Jagdishprasad Gupta, Professor and Head, Department of Gastroenterology, Government Medical College and Super Specialty Hospital, Nagpur, Maharashtra, India, e-mail: sudhirjgupta@gmail.com

Received 7 January 2016; accepted 26 March 2016; published online 20 May 2016

DOI: http://dx.doi.org/10.20524/aog.2016.0049
Malnutrition rate is reported to be $60 \%$ in decompensated cirrhosis and $20 \%$ in compensated cirrhosis [1]. Patients with liver disease frequently suffer from malnutrition and micro/ macronutrient deficiencies. The etiology of malnutrition in cirrhosis is multifactorial including reduced oral intake due to loss of appetite, vomiting, malabsorption and various metabolic abnormalities. In addition alcoholic liver disease patients produce less amount of bile by the affected liver. Bowel edema from hypoalbuminemia and associated chronic pancreatitis in these patients further leads to malnutrition [2]. Index of disease severity for patients with end-stage liver disease has been classified by Child-Turcotte-Pugh (CTP) scoring system. The original classification used nutritional status as a parameter however; it has been replaced by the prothrombin time in newer version [3]. The identification of a novel method for nutritional assessment in patients with cirrhosis is demanding. Conventionally measured parameters of nutritional status, such as body mass index, anthropometry, and biochemical parameters vary with the severity of liver disease and they rarely estimate exact nutritional status [4]. Subjective Global Assessment (SGA) uses clinical criteria 
to determine nutritional status and lack of any objective measurements makes it highly variable in estimating malnutrition [5]. Subjective nature of the many of the classifications used to grade prognosis makes in necessary to have a reliable, reproducible and objective method to mark the presence of malnutrition. Muscle wasting in the cirrhotics is an indirect evidence of the malnutrition. This muscle wasting correlating with the functional loss can be measured with the Handgrip strength (HGS). Association of HGS and health decline has been previously described in the aged population with functional disability [6]. Recently HGS is being regarded as a predictor of postoperative complications, mortality, and functional decline making handgrip dynamometry (HGD) a broader application tool for screening procedure [7].

In view of the above-mentioned lack of efficient tools, the present study was designed to determine the efficacy of HGD as a marker to diagnose nutritional status and to predict the complications and short term mortality in alcoholic liver disease, in addition to CTP class.

\section{Patients and methods}

This was an observational clinical evaluation outcome assessment study at our institute from April 2015 to June 2015. Institutional ethics committee approval was taken prior to the start of the study. All consecutive patients with alcoholic liver disease aged more than 18 years were included in the study. Patients with age $<18$ years; other etiologies of liver disease like HBV and HCV; hepatocellular carcinoma; HIV; chronic renal insufficiency, chronic pancreatitis, cognitive alterations like hepatic encephalopathy: and acute alcohol intoxication on admission were excluded from the study. Alcoholic liver disease was diagnosed based on a combination of thorough history, clinical findings, blood and radiological investigations. All patients underwent the following set of investigations: complete blood count; liver function tests; renal function test; prothrombin time/INR; ultrasound of abdomen; ascitic fluid analysis, and upper gastrointestinal endoscopy. Severity of alcoholic liver disease was assessed by CTP score and model for end -stage liver disease (MELD) score. Nutritional status was assessed with visceral proteins, SGA and HGD.

\section{HGD}

A mechanical HGD (Camrey) (Fig. 1A) was used to assess HGS. Patients sat comfortably on a chair and, after adjusting the handle of the dynamometer, patients were advised to hold the instrument away from body and table. Patients were then advised to hold and compress the handle of dynamometer with as much strength as possible with the dominant hand. Three readings were noted with a gap of more than $30 \mathrm{sec}$. The mean of the 3 recordings was taken into consideration [8]. Normal reference values for handgrip were taken according to age and sex matched data (Fig. 1B). All values were recorded in $\mathrm{kg}$.

Patients were classified prospectively into three groups based on SGA: well nourished- (SGA-A); mild or moderately malnourished- (SGA- B); severely-malnourished (SGA- C). These patients were followed at 3 months for outcome measures and survival. All patients were given a standard treatment and all abstained from alcohol strictly throughout the course of the study and/or death.

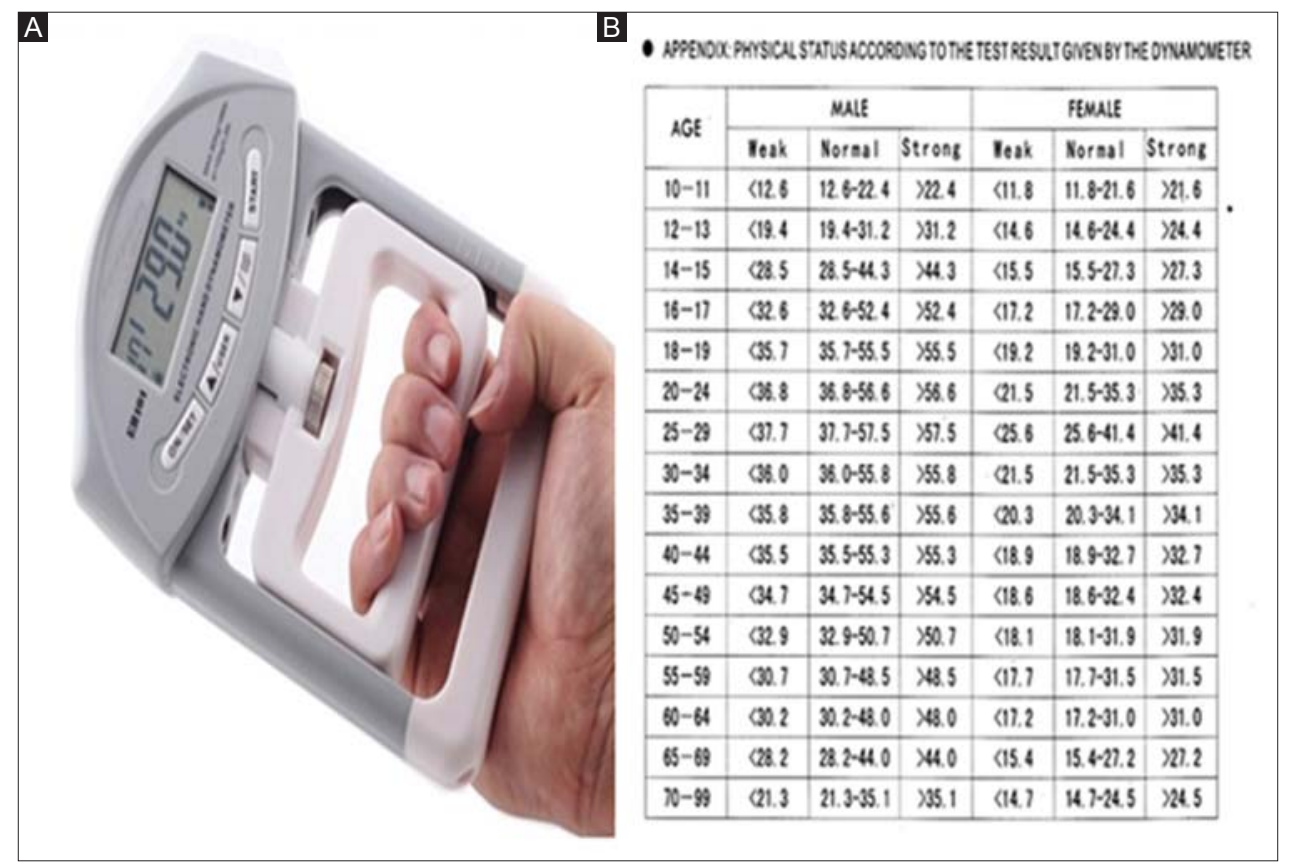

Figure 1 (A) Handgrip dyanamometer; (B) Reference values for handgrip strength 


\section{Statistical analysis}

The statistical analysis performed in the present study is descriptive and inferential. Continuous measurements are presented as mean $\pm \mathrm{SD}$ (min-max) and categorical measurements are presented as number (\%). Significance is assessed at 5\% level of significance. Analysis of variance (ANOVA) has been used to find the significance of study parameters between three or more groups of patients. The significance of study parameters on categorical scale between two or more groups was made by Chi-square/Fisher Exact test. The significance of study parameters on continuous scale between two groups (inter-group analysis) on metric parameters was made by Student's $t$ - test. Multivariate logistic regression analysis was employed to find the predictors of death at 3 months. Kaplan Meier function analysis was employed to determine the mean days of mortality. Pearson factor for correlation between handgrip versus CTP and MELD scores was made.

Data analysis was performed using- the statistical software SAS 9.2, SPSS 15.0, Stata 10.1, MedCalc 9.0.1, Systat 12.0 and R environment ver.2.11.1.

\section{Results}

A total of 80 patients were included in the study. Mean age of the patients was $43.06 \pm 10.03$ years. 69 patients survived and 11 patients died within the study duration of 3 months.

Of the 80 patients enrolled in the study, 20 (25\%) belonged in the well- nourished SGA A group; 51 (63.8\%) in the mild to moderately- malnourished SGA B group; and $9(11.3 \%)$ were in the severely- malnourished SGA C group.
HGS was higher in SGA A $(28.76 \pm 5.48 \mathrm{~kg})$ than SGA B $(22.43 \pm 4.95 \mathrm{~kg})$ and SGA C $(16.78 \pm 3.83 \mathrm{~kg})(\mathrm{P}=<0.001)$. The number of complications including spontaneous bacterial peritonitis,gastrointestinal bleeding, and encephalopathy were: $66.66 \%$ in SGA C group, $20.75 \%$ in SGA B and $10 \%$ in SGA A.

The factors which were significantly different among the 3 nutritional subgroups (SGA A, B, C) are given in Table 1 .

Mean HGS was significantly higher in the survivors

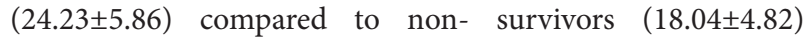
$(\mathrm{P}=0.0011)$. When handgrip was quantified in the deceased and the patients still alive, significant difference was found (Table 2). Mean HGS in deceased patients was $18.04 \pm 4.82 \mathrm{~kg}$ and HGS in patients still alive was $24.23 \pm 5.86 \mathrm{~kg}(\mathrm{P}=0.001)$. Multivariate logistic regression analysis to assess the risk factors for death showed handgrip to be in the suggestive significance range $(\mathrm{P}=0.072)$ (Table 3$)$.

Survival, according to Kaplan Meier curve, is shown in Fig. 2.

Sensitivity of HGS was $88.41 \%$ and specificity was $72.73 \%$ in predicting the short-term mortality when cutoff value for handgrip was taken as $17.05 \mathrm{~kg}$ in ROC curve (Fig. 3).

A strong negative correlation was noted between the HGS and CTP $(\mathrm{r}=-0.606)(\mathrm{P}=<0.0012)$ and also HGS and MELD score $(\mathrm{r}=-0.394)(\mathrm{P}=<0.001)$ assessed by the Pearson correlation coefficient (r) (Fig. 4).

\section{Discussion}

Recognition of malnutrition as a prognostic factor in endstage liver disease is already known but has recently gained more attention. Identifying patients truly malnourished in a large cohort of liver disease requires the use of a simple,

Table 1 Comparison of handgrip and other parameters in the 3 nutritional study subgroups

\begin{tabular}{|c|c|c|c|c|}
\hline \multirow[t]{2}{*}{ Variables } & \multicolumn{3}{|c|}{ SGA class $($ mean \pm SD) } & \multirow[t]{2}{*}{$\mathrm{P}$ value } \\
\hline & A $(n=20)$ & $B(n=51)$ & $C(n=9)$ & \\
\hline Age in years & $44.40 \pm 8.85$ & $42.08 \pm 10.85$ & $45.67 \pm 7.33$ & 0.489 \\
\hline Hemoglobin (g/dL) & $9.94 \pm 2.89$ & $8.30 \pm 2.45$ & $8.06 \pm 1.64$ & $0.039^{*}$ \\
\hline Platelet count $/ \mathrm{mm}^{3}$ & $140200.00 \pm 93951.05$ & $114235.29 \pm 71109.94$ & $51777.78 \pm 15155.67$ & $0.015^{\star}$ \\
\hline Total bilirubin (mg/dL) & $1.71 \pm 1.31$ & $4.39 \pm 5.98$ & $9.07 \pm 6.6$ & $0.004^{* *}$ \\
\hline Total protein $(\mathrm{g} / \mathrm{dL})$ & $6.66 \pm 0.66$ & $6.42 \pm 0.83$ & $5.71 \pm 0.74$ & $0.013^{*}$ \\
\hline Serum albumin (g/dL) & $3.23 \pm 0.56$ & $2.92 \pm 0.58$ & $2.41 \pm 0.29$ & $0.002^{\star *}$ \\
\hline INR & $1.17 \pm 0.19$ & $1.62 \pm 1.31$ & $2.20 \pm 1.08$ & $0.067^{+}$ \\
\hline Blood urea (mg/dL) & $29.15 \pm 20.93$ & $31.84 \pm 23.11$ & $43.89 \pm 41.51$ & 0.333 \\
\hline MDF & $10.87 \pm 10.90$ & $25.14 \pm 35.42$ & $54.37 \pm 32.49$ & $0.003^{* *}$ \\
\hline MELD & $10.50 \pm 2.67$ & $15.40 \pm 7.21$ & $24.84 \pm 11.02$ & $<0.001^{\star *}$ \\
\hline Handgrip (kg) & $28.76 \pm 5.48$ & $22.43 \pm 4.95$ & $16.78 \pm 3.83$ & $<0.001^{\star *}$ \\
\hline
\end{tabular}

${ }^{+}$Suggestive significance $(0.05<\mathrm{P}<0.10) ;{ }^{*}$ moderately significant $(0.01<\mathrm{P} \leq 0.05) ;{ }^{* *}$ strongly significant $(\mathrm{P} \leq 0.01)$

SGA, subjective global assessment; INR, international normalized ratio; MDF, Maddrey's discriminant function; MELD, model for end stage liver disease 
reliable and reproducible method. The reported prevalence of malnutrition in CTP class A is around 25\% [9]. Use of simple bedside methods such as SGA or anthropometry to identify patients at high risk of malnutrition was advised by the European Society for Clinical Nutrition and Metabolism (ESPEN) guidelines [10]. Many of the tests used to measure the nutritional status in cirrhotics yielded diverse results including some tests underestimating the malnutrition like body mass index and some overestimating the malnutrition like serum albumin. Prevalence of sarcopenia in cirrhotics has been reported around $60 \%$ in recently published studies [11].

In this regard HGS measurement offers the advantage

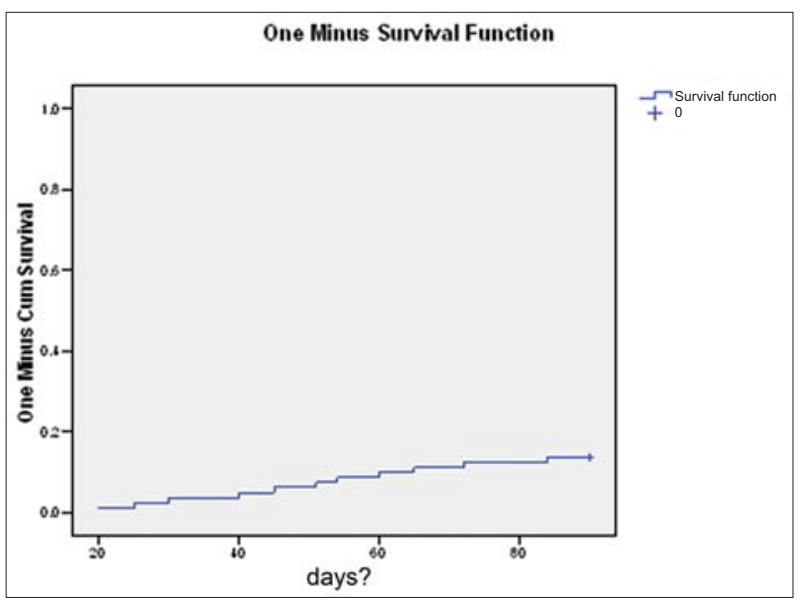

Figure 2 Kaplan Meier function analysis. Mean Estimate $=84.45$, $\mathrm{SE}=1.75$, Lower $\mathrm{CI}=81.03$, Upper $\mathrm{CI}=87.87$

Table 2 Handgrip levels of patients studied in relation to outcome at 3 months

\begin{tabular}{lccc}
\hline \multirow{2}{*}{ Handgrip } & \multicolumn{2}{c}{ Outcome at 3 months } & \multirow{2}{*}{ Total } \\
\cline { 2 - 3 } & Deceased & Alive & \\
\hline$<20$ & $8(72.7 \%)$ & $16(23.2 \%)$ & $24(30 \%)$ \\
\hline $20-30$ & $3(27.3 \%)$ & $41(59.4 \%)$ & $44(55 \%)$ \\
$>30$ & $0(0 \%)$ & $12(17.4 \%)$ & $12(15 \%)$ \\
\hline Total & $11(100 \%)$ & $69(100 \%)$ & $80(100 \%)$ \\
\hline Mean \pm SD & $18.04 \pm 4.82$ & $24.23 \pm 5.86$ & $23.38 \pm 6.09$ \\
\hline
\end{tabular}

$\mathrm{P}=0.001^{*}$, significant; Student's $t$-test over other tests in terms of reproducibility and objectivity in predicting the mortality.

Functional loss in malnourished patients is attributed to decreased body cell mass. Therefore, use of a technique capable of assessing functional capacity is of paramount importance [12] and helps detect malnutrition at an early stage. To satisfy these requirements, HGD is a reliable method which can assess the muscle strength and functional capacity [13]. HGD is tool for the nutritional screening and has been used in diagnosis of malnutrition in healthy populations [14] and hospitalized individuals [12]. HGS was used as a proxy for muscle strength in many studies as mentioned in previous literature [15]. In the study of Alvares-da-Silva et al, HGD was reported as a simple method to detect malnutrition among patients who are at nutritional risk, and after comparison with SGA they have concluded that handgrip examination can be considered a reliable method to identify malnutrition and is easy to perform [16].

HGS testing is increasingly used in the assessment of sarcopenia [17], frailty and under nutrition in hospitalized older people.

The progression of malnutrition in cirrhotics can be predicted by decreased HGS. Álvares-da-Silva et al [18] observed that the HGD presents the capacity to detect $100 \%$ of the malnutrition cases. Norman et al [19] mentioned the best use of HGD to identify the nutritional risk before the end stage of malnutrition. HGS is a crucial nutritional parameter in detecting body cell mass depletion which is associated with adverse clinical outcomes in patients with end-stage liver disease. HGS is a better predictor than standard SGA or the prognostic nutritional index in predicting the adverse effects related to malnutrition.

Our study has shown more complications (spontaneous bacterial peritonitis, gastrointestinal bleeding and hepatic encephalopathy) in the severely-malnourished SGA C group (66.66\%) compared to SGA B group (20.75\%) and SGA A group $(10 \%)(\mathrm{P}=0.045)$ Other recent studies have also shown similar results with complication rates around $65.6 \%$ in malnourished cirrhotic patients as compared to the only $5.8 \%$ in those without malnutrition [16].

In our study handgrip was strongest in CTP class A followed by CTP class B and C, suggesting the inverse relation between HGS and CTP score. Abbott et al found an association of reduced muscle mass with higher CTP score and showed an increase in total morbidity as well

Table 3 Multivariate logistic regression analysis to assess the risk factors for death

\begin{tabular}{|c|c|c|c|c|c|c|c|}
\hline \multirow[t]{2}{*}{ Variables } & \multicolumn{5}{|c|}{ Logistic regression results to predict the death } & \multicolumn{2}{|c|}{$95 \% \mathrm{CI}$} \\
\hline & Logit co-efficient & SE & Wald & $P$ value & Adj OR & Lower & Upper \\
\hline Alcohol ingestion duration & 0.05 & 0.10 & 0.22 & 0.643 & 1.05 & 0.86 & 1.27 \\
\hline CTP score & -0.41 & 0.31 & 1.74 & 0.187 & 0.66 & 0.36 & 1.22 \\
\hline MDF & -0.04 & 0.02 & 2.12 & 0.146 & 0.96 & 0.92 & 1.01 \\
\hline Handgrip & 0.24 & 0.13 & 3.25 & $0.072^{+}$ & 1.27 & 0.98 & 1.64 \\
\hline
\end{tabular}




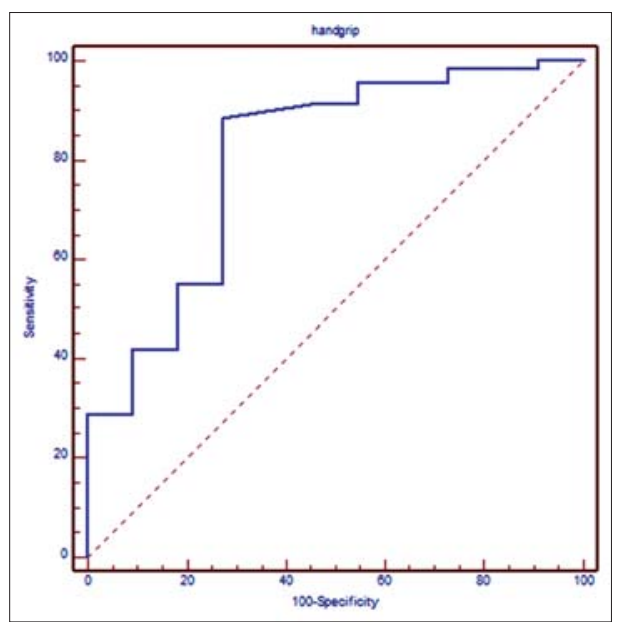

Figure 3 ROC for handgrip strength. Sensitivity $=88.41 \%$, Specificity $=72.73 \%$, Cut-off $=<17.5$, AUROC $=0.805$

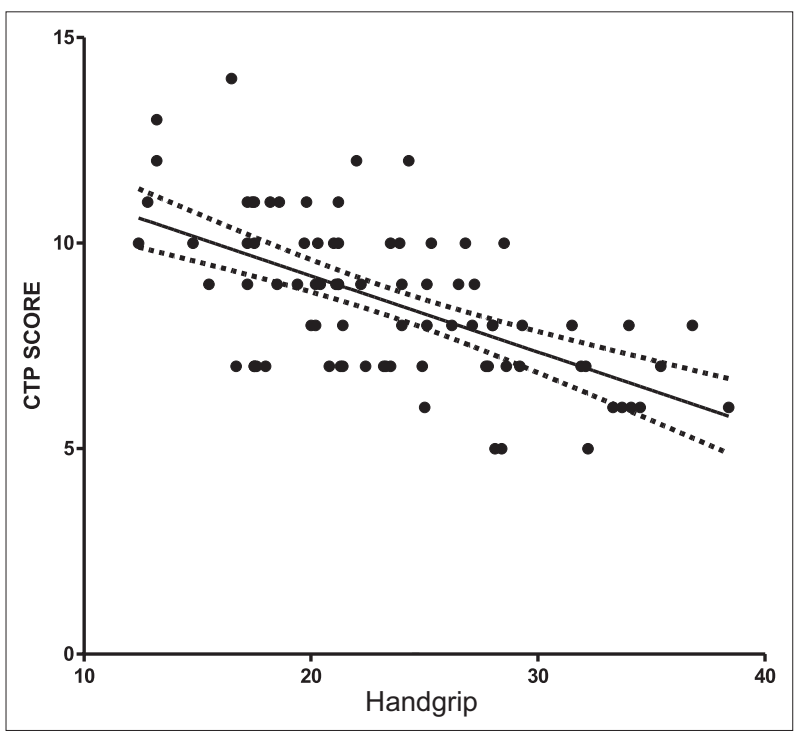

Figure 4 Correlation between the Child-Turcotte-Pugh (CTP) score and handgrip strength

as early morbidity in post-liver transplantation patients. Reduced HGS was associated with increased duration of hospitalization, increased postoperative complications, higher numbers of rehospitalization and decreased physical capability [4].

Mortality in our study was significantly higher in the severely- malnourished group (SGA C) compared to the mild to moderately -malnourished group (SGA B) and the well nourished group (SGA A). Low HGS predicts increased risk of all-cause mortality reported by many epidemiological studies [20]. Muscle function and/or mass reacts early in response to nutritional deprivation. HGD has become a popular modality of nutritional status assessment and is increasingly being used as outcome variable in nutritional assessment programs [21].

\section{Summary Box}

\section{What is already known:}

- Malnutrition in alcoholic liver disease is associated with many adverse effects and signifies poor prognosis

- Current markers of malnutrition predict malnutrition variably and it is difficult to rely on any of them

- Subjective global assessment (SGA) has many limitations and its use for nutritional assessment is error prone

- Handgrip strength is already being evaluated in the elderly and hospitalized patients

\section{What the new findings are:}

- We studied the utility of handgrip dynamometry in predicting short term mortality in alcoholic liver disease and found it to be a highly sensitive method

- Impaired handgrip strength was associated with many complications and poor short term survival

- Handgrip strength offers many advantages over SGA e.g. simplicity, objectivity and easy reproducibility

- Handgrip dynamometry can be used reliably to identify patients with poor nutrition and to predict their prognosis

Our study showed that weak handgrip was associated with severe malnutrition which in turn predicts more complications and poor prognosis in alcoholic liver disease patients.

The sensitivity of HGS in predicting short- term mortality was $88.41 \%$ in our study which can be considered as a test with a high sensitivity and might have a role in the screening for malnutrition.

Our study has a number of limitations. Subjective components in the SGA may differ amongst individuals so classifying patients according to SGA class is difficult. On multivariate analysis handgrip showed a trend towards significance (suggestive significance) but not statistical significance. Futures larger studies and with a longer duration are required to accurately validate the accuracy of HGD in predicting mortality.

In conclusion, in addition to the conventional parameters used to measure malnutrition in cirrhotics, HGS acts as a surrogate marker of malnutrition to predict the risk of complications and short- term mortality. Easy availability, reproducibility, high sensitivity and objective nature of the HGD compared to SGA, are promising factors to render it a risk stratifying method to judge nutritional status in cirrhotics so as to take necessary action. 


\section{References}

1. Coltorti M, Del Vecchio-Blanco C, Caporaso N, Gallo C, Castellano L. Liver cirrhosis in Italy. A multicentre study on presenting modalities and the impact on health care resources. National Project on Liver Cirrhosis Group. Ital J Gastroenterol 1991;23:42-48.

2. Henkel AS, Buchman AL. Nutritional support in patients with chronic liver disease. Nat Clin Pract Gastroenterol Hepatol 2006;3:202-209.

3. Pugh RN, Murray-Lyon IM, Dawson JL, Pietroni MC, Williams R. Transection of the oesophagus for bleeding oesophageal varices. $\mathrm{Br}$ J Surg 1973;60:646-649.

4. Abbott WJ, Thomson A, Steadman C, et al. Child-Pugh class, nutritional indicators and early liver transplant outcomes. Hepatogastroenterology 2001;48:823-827.

5. Detsky AS, McLaughlin JR, Baker JP, et al. What is subjective global assessment of nutritional status? JPEN J Parenter Enteral Nutr 1987;11:8-13.

6. Giampaoli S, Ferrucci L, Cecchi F, et al. Hand-grip strength predicts incident disability in non-disabled older men. Age Ageing 1999;28:283-288.

7. Bohannon RW. Dynamometer measurements of hand-grip strength predict multiple outcomes. Percept Mot Skills 2001;93:323-328.

8. Roberts HC, Denison HJ, Martin HJ, et al. A review of the measurement of grip strength in clinical and epidemiological studies: towards a standardised approach. Age and Ageing 2011;40:423-429.

9. Guglielmi FW, Panella C, Buda A, et al. Nutritional state and energy balance in cirrhotic patients with or without hypermetabolism. Multicentre prospective study by the 'Nutritional Problems in Gastroenterology' Section of the Italian Society of Gastroenterology (SIGE). Dig Liver Dis 2005;37:681-688.

10. Plauth M, Merli J, Kondrup A, et al. Espen Guidelines for nutrition in liver disease and transplantation. Clin Nutr 1997;16:43-55.
11. Hanai T, Shiraki M, Nishimura K, et al. Sarcopenia impairs prognosis of patients with liver cirrhosis. Nutrition 2015;31:193-199.

12. Pham NV, Cox-Reijven PL, Wodzig WK, Greve JW, Soetes PB. SGA and measures for muscle mass and strength in surgical Vietnamese patients. Nutrition 2007;23:283-291.

13. Leal VO, Mafra D, Fouque D, Anjos LA. Use of handgrip strength in the assessment of the muscle function of chronic kidney disease patients on dialysis: a systematic review. Nephrol Dial Transplant 2010;26:1354-1360.

14. Schlüssel MM, Anjos LA, Vasconcellos MT, Kac G. Reference values of handgrip dynamometry of healthy adults: a populationbased study. Clin Nutr 2008;27:601-607.

15. Lauretani F, Russo CR, Bandinelli S, et al. Age-associated changes in skeletal muscles and their effect on mobility: an operational diagnosis of sarcopenia. J Appl Physiol 2003;95:1851-1860.

16. Alvares-da-Silva NR, Reverbel da Silveira T. Comparison between handgrip strength, subjective global assessment, and prognostic nutritional index in assessing malnutrition and predicting clinical outcome in cirrhotic outpatients. Nutrition 2005;21:113-117.

17. Cruz-Jentoft AJ, Baeyens JP, Bauer JM, et al. Sarcopenia: European consensus on definition and diagnosis: report of the European Working Group on Sarcopenia in Older People. Age Ageing 2010;39:412-423.

18. Álvares-da-Siva MR, Silveira TR. Hand-grip strength or muscle mass in cirrhotic patients: who is the best? Nutrition 2006;22:218-219.

19. Norman K, Smoliner C, Valentini L, Lochs H, Pirlich M. Is bioelectrical impedance vector analysis of value in the elderly with malnutrition and impaired functionality? Nutrition 2007;23:564-569.

20. Gale CR, Martyn CN, Cooper C, Sayer AA. Grip strength, body composition, and mortality. Int J Epidemiol 2007;36:228-235.

21. Norman K, Stobäus N, Gonzalez MC, Schulzke JD, Pirlich M. HGS: outcome predictor and marker of nutritional status. Clin Nutr 2011;30:135-142. 